\title{
Clock genes and sleep
}

\author{
Dominic Landgraf • Anton Shostak • Henrik Oster
}

Received: 21 February 2011 /Revised: 8 July 2011 /Accepted: 22 July 2011 / Published online: 11 August 2011

(C) Springer-Verlag 2011

\begin{abstract}
In most species - from cyanobacteria to humans endogenous clocks have evolved that drive 24-h rhythms of behavior and physiology. In mammals, these circadian rhythms are regulated by a hierarchical network of cellular oscillators controlled by a set of clock genes organized in a system of interlocked transcriptional feedback loops. One of the most prominent outputs of the circadian system is the synchronization of the sleep-wake cycle with external (day-) time. Clock genes also have a strong impact on many other biological functions, such as memory formation, energy metabolism, and immunity. Remarkably, large overlaps exist between clock gene and sleep (loss) mediated effects on these processes. This review summarizes sleep clock gene interactions for these three phenomena, highlighting potential mediators linking sleep and/or clock function to physiological output in an attempt to better understand the complexity of diurnal adaptation and its consequences for health and disease.
\end{abstract}

Keywords Circadian clock · Clock genes $\cdot$ Sleep ·

Metabolism $\cdot$ Immunity $\cdot$ Memory

\section{Introduction}

Almost 40 years have passed since the first clock gene, period, was discovered by Ronald Konopka and Seymour

D. Landgraf and A. Shostak contributed equally to this work.

This article is published as part of the Special Issue on Sleep.

D. Landgraf $\cdot$ A. Shostak $\cdot$ H. Oster $(\bowtie)$

Circadian Rhythms Group,

Max Planck Institute for Biophysical Chemistry,

Am Faßberg 11,

37077 Göttingen, Germany

e-mail: henrik.oster@mpibpc.mpg.de
Benzer in a forward genetic screen on fruit flies [75]. Starting from this landmark finding, $N$-ethyl- $N$-nitrosourea (ENU) mutagenesis phenotypic screens became a powerful tool to unravel the genetic basis of circadian rhythms. In the mid-1990s, the first mammalian clock gene, circadian locomotor output cycles kaput (Clock), was identified and cloned by Martha Vitaterna in the lab of Joseph Takahashi $[71,156]$. In mammals - as in most organisms studied so far-circadian rhythms are controlled by a set of clock genes forming a network of positive and negative autoregulatory feedback loops $[57,120]$. These clock genes are expressed in most tissues. A circadian pacemaker located in the suprachiasmatic nuclei $(\mathrm{SCN})$ of the hypothalamus is reset by external light stimuli and synchronizes peripheral oscillators throughout the body with each other and with the light-dark cycle via humoral, neuronal, and behavioral cues [120]. At its heart, the cellular circadian clockwork consists of a main (or core) and an auxiliary (or accessory) transcriptional-translational feedback loop (TTL; Fig. 1, left side). The former is composed of the positive components brain and muscle ARNT-like 1 (BMAL1 or ARNTL), CLOCK and neuronal PAS domain protein 2 (NPAS2), as well as the negative components CRYPTOCHROME 1/2 (CRY1/2) and PERIOD 1-3 (PER1-3). BMAL1, CLOCK, and NPAS2 are members of the basic helix-loop-helix (bHLH) Per-Arnt-Sim (PAS) family of transcription factors. In the SCN, CLOCK/NPAS2 and BMAL1 form heterodimers that bind to specific circadian E-box elements on the promoters of their targets, thereby activating Cry and Per transcription during the (subjective) day. In the late afternoon, PER and CRY protein levels reach a critical concentration and, now forming complexes themselves, translocate into the nucleus. There, they interact with CLOCK/NPAS2-BMAL1 and, by repressing the transcription of their own genes, form a negative feedback loop. Progressive degradation of negative 


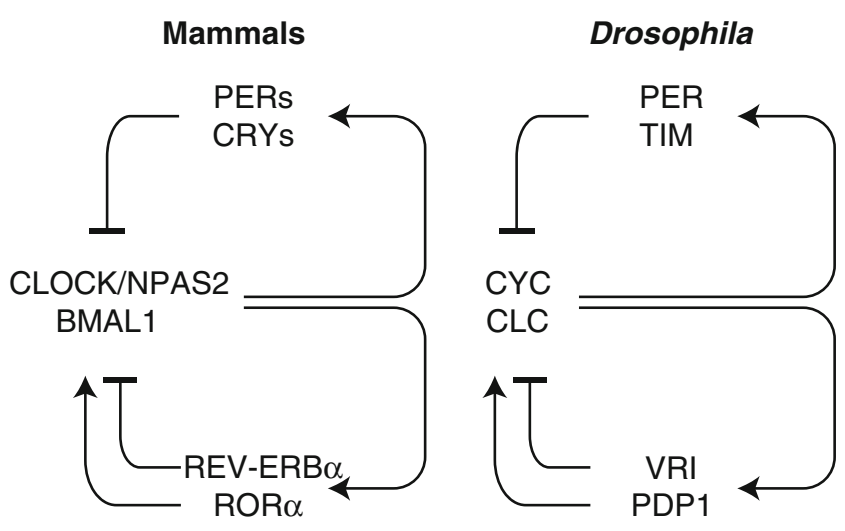

Fig. 1 Transcriptional-translational feedback loops regulate cellular circadian rhythms in mammals and flies. Both vertebrate and invertebrate clockworks are based on similar mechanistic concepts and share a number of genetic components. In mammals (left), a core loop is composed of PER and CRY proteins that inhibit their own transcription by inhibition of CLOCK (NPAS2)/BMAL1. An accessory loop involves REV-ERB $\alpha$ and $\operatorname{ROR} \alpha$ that regulate Bmall transcriptional rhythms. In Drosophila (right), CLC/CYC activate PER/TIM that feedback on CLC/CYC activity. VRI and PDP1 form an accessory loop that regulates $\mathrm{Clc}$ transcriptional rhythms

regulators towards the end of the subjective night starts a new cycle by the re-activation of Per/Cry transcription. Posttranscriptional modifications are heavily involved in clock oscillations and impart precision and robustness to the TTL. In particular, members of the casein kinase family (e.g., $\mathrm{CKI} \varepsilon, \mathrm{CKI} \delta$ ) are known to phosphorylate PER proteins at conserved residues and promote degradation, thereby delaying PER nuclear entry $[48,83]$. The auxiliary loop comprises two genes of the orphan nuclear receptor family, Rev-erb $\alpha$ (Nrldl) and Ror $\alpha$ (Rora). REV-ERB $\alpha$ and ROR $\alpha$ repress or activate, respectively, the transcription of genes with ROR elements in their promoters, such as Bmall and Npas2. Rev-erb $\alpha$ and Ror $\alpha$ are considered to be dispensable for cellular rhythm generation, yet they were shown to regulate phasing and amplitude of clock gene expression rhythms [116, 127]. Further ancillary loops have been described. The CLOCK/NPAS2-BMAL1-regulated bHLH transcription factors DEC1 (BHLHE40) and DEC2 (BHLHE41) were shown to bind E-box elements and modulate CLOCK/NPAS2/BMAL1-driven circadian transcription [62, 124]. Another TTL involves the two transcription factors $\mathrm{D}$-site albumin promoter binding protein and E4BP4 (NFIL3) that compete for binding of D-boxes, a third circadian regulatory DNA motif, at the promoters of Per1-3, Rev-erb $\alpha$, Ror $\alpha$, and various clockcontrolled genes (CCGs) [90, 101]. Similarly, in Drosophila, CYCLE (CYC) and CLOCK (CLK), the orthologs of BMAL1 and CLOCK, form heterodimers and activate transcription of the circadian repressor genes Timeless (Tim) and Period (Per) via E-boxes (Fig. 1, right side). TIM is a substitute for mammalian CRYs as the major core TTL inhibitor, whereas in the fly, CRY functions primarily as a photoreceptor and helps to synchronize the clock to the lightdark cycle [142]. TIM/PER complexes are transported to the nucleus to repress CYC/CLK-mediated transcription [56]. DOUBLETIME, a homolog of casein kinase I, phosphorylates PER, assigning it for sequestration via the proteasomal pathway [72, 117]. Similar to mammals, the fly clock contains at least one auxiliary loop consisting of VRILLE (VRI) and PAR-domain protein-1 (PDP1). The former inhibits, whereas the latter activates Clk transcription [26].

\section{Interaction between circadian and homeostatic sleep components}

The current model predicts that sleep is regulated by two principle mechanisms $[13,27]$. The first, termed process $c$, determines the appropriate timing of sleep. Nocturnal animals experience sleep mostly during the day, while diurnal species such as humans rest predominantly during the night. The SCN, as master circadian pacemaker, sends projections to important sleep regulatory nuclei such as the ventrolateral pre-optic area, the dorsomedial nucleus of the hypothalamus, and the hypocretin/orexin neurons of the lateral hypothalamus. Process $\mathrm{c}$ is complemented by a homeostatically controlled sleep drive, process $\mathrm{s}$, which builds up an increased need for sleep in response to extended wake periods, independent of the time of day. So far, the anatomical substrate of process $\mathrm{s}$ remains elusive. Of note, sleep in mammals and birds is quantified primarily by electro-encephalography (EEG). In contrast, characterization of sleep in insects, where rhythm and homeostasis appear to be as robust as in mammals, relies mostly on measurements of rest/activity periods and arousal thresholds [60, 131]. SCN-lesioned rats and mice show disrupted sleep timing and consolidation, though the overall time spent asleep each day and the delta response to sleep deprivation remain uncompromised [67, 100, 147]. Previous studies conveyed on humans under forced desynchrony protocols demonstrated that slow wave activity was largely independent of internal circadian phase, though distribution of REM sleep and spindle activity in non-rapid eye movement (NREM) sleep correlated with body temperature rhythms [34]. At the same time, homeostatic sleep components can modify circadian pacemaker function. Sleep states affect activity of SCN neurons with decreasing firing rates during NREM phase and after sleep deprivation [30, 31]. Moreover, prolonged awaking effects the expression of clock genes in the cerebral cortex, upregulating both of Per 1 and Per $2[161,162]$. This body of evidence suggests that the circadian clock regulates sleep-wake timing and opposes process $\mathrm{s}$ in order to gate consolidated bouts of sleep and waking. 


\section{Clock genes and sleep timing}

In humans, naturally occurring polymorphisms in clock genes correlate with early or late chronotype. A PER3 gene length polymorphism is linked to extreme diurnal preferences [4]. The longer allele, which carries five repetitions $\left(P E R 3^{5 / 5}\right)$ of a variable number tandem repeat, is associated with early morning type, whereas the shorter allele $\left(P E R 3^{4 / 4}\right)$ correlates with eveningness and delayed sleep phase syndrome (DSPS). Recently, it was demonstrated that a polymorphism in the PER3 promoter is also associated with DSPS [6]. Similar correlations of polymorphic alleles with diurnal preferences are observed for PERI and the 5'untranslated region of PER2 [18, 19].

Circadian control of sleep can be better demonstrated on disorders associated with extremely shifted sleep-wake time. In familiar advanced sleep phase syndrome (FASPS), genetic studies identified mutations in the PER2 (S662G) and CKI $\delta$ (T44A) genes in some families [148, 164]. Remarkably, both mutations affect an evolutionary conserved process, the phosphorylation of the PER2 protein by CKI. Xu et al. [164] have shown that a single amino acid substitution (T44A) in the human CKI $\delta$ protein decreases its enzymatic activity in vitro. The corresponding mutation, when reproduced in mice, causes a shortened circadian period length, which is consistent with symptoms of FASPS patients. Surprisingly, a miss-sense mutation in the same conserved residue of the Drosophila CKI ortholog Dbt leads to an increase in the free-running period [164], as would be expected from DSPS patients. These findings highlight the different organization of circadian/sleep regulatory mechanisms in insects and mammals, despite the fact that individual components share a great similarity between vertebrate and invertebrate systems (see also Fig. 1). In another study, transgenic mice expressing human PER2 with the S662G mutation on a Per2-deficient background display a shorter period resembling humans with FASPS. Conversely, an aspartate substitution at the same residue (S662D), mimicking a constitutively phosphorylated state, correlates with a longer period [165].

\section{Clock genes and sleep homeostasis}

A number of recent studies suggest that clock genes, besides regulating circadian sleep-wake timing, also contribute to sleep homeostatic control (reviewed in [47] and summarized in Table 1). Naylor et al. [105] demonstrated that mutations in Clock have effects on a variety of sleepwake parameters in the mouse. Clock mutant animals show a reduction in total sleep time (around $2 \mathrm{~h}$ ) under light/dark (LD) conditions, mostly due to reduced NREM sleep. In constant darkness (DD), homozygous mutants spend more time of their circadian cycle awake, mostly sacrificing NREM sleep, even when the results are normalized to their longer endogenous circadian period of $28.8 \mathrm{~h}$. The response to sleep deprivation is also altered in these mice with decreased REM sleep rebounds, though the effects on

Table 1 Clock gene mutant mice with sleep abnormalities

\begin{tabular}{|c|c|c|c|c|c|c|c|}
\hline \multirow[t]{2}{*}{ Mouse mutant } & \multirow{2}{*}{$\begin{array}{l}\text { Circadian } \\
\text { phenotype }\end{array}$} & \multicolumn{6}{|l|}{ Sleep phenotype } \\
\hline & & $\begin{array}{l}\text { Sleep amount, } \\
\text { light/dark phase }\end{array}$ & $\begin{array}{l}\text { REM, light/ } \\
\text { dark phase }\end{array}$ & $\begin{array}{l}\text { NREM, light/ } \\
\text { dark phase }\end{array}$ & $\begin{array}{l}\text { Delta power in } \\
\text { NREM, light/ } \\
\text { dark phase }\end{array}$ & $\begin{array}{l}\text { Response to sleep } \\
\text { deprivation }\end{array}$ & Reference \\
\hline Bmall $^{-/-}$ & Arrhythmic & Normal/elevated & Normal/elevated & Normal/elevated & Elevated/reduced & $\begin{array}{l}\text { Attenuated NREM/ } \\
\text { REM }\end{array}$ & {$[81]$} \\
\hline$N p a s 2^{-1-}$ & Short period & Normal/reduced & Normal/reduced & Normal/reduced & Normal/normal & $\begin{array}{l}\text { Attenuated NREM, } \\
\text { reduced delta } \\
\text { power }\end{array}$ & {$[39,46]$} \\
\hline Clock $^{\Delta 19}$ & Long period & Reduced/reduced & Normal/normal & Reduced/reduced & - & Attenuated REM & {$[105]$} \\
\hline $\operatorname{Per} 1 / 2^{m / m}$ & Arrhythmic & Reduced/normal & Normal/normal & Reduced/normal & Normal/normal & $\begin{array}{l}\text { Increased delta } \\
\text { power }\end{array}$ & [135] \\
\hline $\operatorname{Cry} 1 / 2^{--}$ & Arrhythmic & Normal/elevated & Reduced/elevated & Normal/elevated & Elevated/elevated & $\begin{array}{l}\text { Attenuated NREM/ } \\
\text { REM, reduced } \\
\text { delta power }\end{array}$ & {$[161]$} \\
\hline$D b p^{-/-}$ & Short period & Normal/normal & Reduced/normal & Normal/normal & Normal/reduced & Attenuated REM & {$[45]$} \\
\hline $\operatorname{Dec} 2^{P 385 R}$ & Normal & Reduced/normal & Reduced/normal & Reduced/normal & Normal/normal & $\begin{array}{l}\text { Attenuated NREM/ } \\
\text { REM, reduced } \\
\text { delta power }\end{array}$ & [59] \\
\hline$P K 2^{-/-}$ & $\begin{array}{l}\text { Attenuated } \\
\text { amplitude }\end{array}$ & Reduced/normal & Normal/elevated & Reduced/normal & Normal/normal & $\begin{array}{l}\text { Attenuated NREM/ } \\
\text { REM, reduced } \\
\text { delta power }\end{array}$ & {$[65]$} \\
\hline Vipr $2^{-/}$ & Arrhythmic & Reduced/elevated & Reduced/elevated & Reduced/elevated & Normal/normal & - & [133] \\
\hline
\end{tabular}


NREM and total sleep are unchanged [105]. Gene association studies performed on two independent populations of humans report links between sequence variants of CLOCK and sleep duration [1]. In the clock machinery, Npas2 acts as a functional paralog of Clock, yet their expression in the brain rarely overlaps [2, 50, 71]. Consistent with this, Npas2-deficient mice show about $25 \%$ reduction in NREM and REM sleep as well as reduced sleep consolidation [39]. Subjected to $8 \mathrm{~h}$ of prolonged waking, Npas 2 mutants display a smaller compensatory increase in NREM sleep and in delta activity [46].

Bmall-deficient mice are to date the only reported mouse strain in which the deletion of a single gene totally disrupts circadian molecular and behavioral rhythms [17]. Consistent with their arrhythmic behavior, homozygous $\mathrm{Bmall}^{-/-}$mice show attenuated sleep-wake rhythms and increased sleep fragmentation. In contrast to Clock mutants, Bmal1 ${ }^{-/}$animals exhibit longer REM and NREM periods under LD and DD conditions. Furthermore, during the light phase delta power is constantly high, indicating that these animals are persistently under elevated sleep pressure. Paradoxically, when actively sleep deprived, Bmall mutants show an attenuated homeostatic response. This might be due to a lesser amount of sleep lost during sleep restriction when compared to wild-type animals [81].

Mice lacking both Cryl and Cry2 genes are frequently used as a genetic model of circadian arrhythmicity [152, 157]. In $C r y 1 / 2^{-/}$mice, sleep parameters do not differ between light and dark phases, indicating a non-circadian distribution of sleep. Both NREM sleep and EEG delta power are increased during the light phase, and Cryl/2 mutants also show attenuated responses to sleep restriction [161]. Of note, single Cryl or Cry2 knockouts do not display any significant differences in sleep parameters consistent with their - at least partially - redundant role in the circadian clock [162]. Similarly, Per gene mutations have only modest effects on sleep homeostasis. Studies done on both Per1 and Per2 single mutant mice reveal altered 24-h distribution of sleep but normal responses to sleep deprivation [76, 135, 162]. However, behaviorally arrhythmic Perl/2 double mutant animals show decreased REM and NREM sleep periods during the light phase and moderately increased delta power after prolonged waking [135]. Remarkably, in rats, expression of Per1 in the dorsomedial SCN was correlated with timing of REM sleep occurrence, pointing to a function of the central pacemaker itself in sleep architecture regulation [84]. In humans, the PER3 gene also plays a role in sleep homeostasis. Individuals bearing the gene length variant $P E R 3^{5 / 5}$ show longer durations of NREM sleep bouts, higher delta power, and an exaggerated response to sleep deprivation [155].
In Drosophila, mutants of mammalian clock gene orthologs also exhibit profound changes in sleep homeostasis. In particular $\mathrm{clk}^{j r k}$, $\mathrm{per}^{01}$, and $\mathrm{tim}^{01}$ flies show increased sleep rebounds after 7, 9, and $12 \mathrm{~h}$ of sleep deprivation and recover $100 \%$ (compared to $30-40 \%$ in wild-type Canton-S flies) of sleep lost within 12 h [132]. In turn, more tremendous sleep rebound and even lethality in response to $12 \mathrm{~h}$ of sleep deprivation have been observed in $c y c^{01}$ mutants [132]. Interestingly, this phenotype differs between genders with stronger effects seen in females [61].

Dbp knockout mice were the first animal model investigated for the role of clock genes in sleep homeostasis [45]. In constant darkness, Dbp-deficient mice exhibit a slightly shorter free-running period and decreased overall activity [90]. On EEG recordings $D b p$ mutants show reduced REM sleep during the light phase as well as less delta power activity in the dark. After $6 \mathrm{~h}$ of sleep restriction, significantly attenuated REM responses are observed [45]. In a recent study, $\mathrm{He}$ and co-workers [59] found $D E C 2$ to regulate sleep length in humans. They identified a miss-sense mutation in the human $D E C 2$ gene that is associated with a sleep phenotype (6 vs. $8 \mathrm{~h}$ sleep duration in control subjects). When this point substitution is reproduced in mice, it decreases total sleep time via both REM and NREM without affecting circadian period [59]. A targeted deletion of $D e c 2$, however, does only produce a mild sleep phenotype in mice [59]. Interestingly, in the fly homolog of DEC2, CLOCKWORK ORANGE (CWO) [87], the affected amino acid residue (P385) is not conserved, but flies expressing a mutant mouse Dec2 show a similar sleep phenotype [59].

Of note, some of the genes known to mediate transcriptional output from the circadian clock machinery have also been implicated in sleep regulation. For instance, targeted deletion of Prokineticin2 (Pk2), encoding a peptide secreted from the SCN and critical for the maintenance of robust circadian behavioral rhythms $[24,86]$, produces profound alterations in sleep homeostasis. Pk2 mutants show a $20 \%$ reduction in total sleep time, mostly due to a decrease in NREM sleep in the light phase, whereas REM sleep is increased. Delta power and REM sleep rebound after sleep deprivation are also attenuated in these mice [65]. A recent study on the VPAC2 subtype of the VIP receptor (VIPR2) implicated in the coupling of cellular oscillators within the SCN pacemaker demonstrates the significance of synchronization of electrical activity in SCN neurons on sleep regulation. Consistent with locomotor activity data, an attenuated diurnal rhythm of sleep and wakefulness is seen in Vipr2-deficient mice, although total sleep time and other homeostatic parameters are not affected [58, 133]. Interestingly, in flies, a disruption of the VIP analog neuropeptide pigment dispersing factor (PDF) increases sleep and causes reduced responsiveness to external stimuli [25]. Together, 
these studies clearly show that circadian and homeostatic regulatory circuits show a high degree of interaction. It remains unclear, however, how this cross-talk is mediated at the molecular level and which neuronal circuits are involved.

\section{Clock genes and sleep-associated functions}

Clock genes do not only influence sleep architecture and quality. They might also be involved in sleep and sleepcorrelated functions within an organism [51]. In many cases, clock gene mutations and sleep disorders share the same symptoms and phenotypes. Sleep loss, for example, has been correlated to numerous metabolic symptoms, which are also observed in circadian clock-deficient animal models. Clock genes affect synaptic plasticity in learning and memory formation and modulate immune functions during the course of the day. In the same way, sleep-or the lack thereof-has a strong impact on these processes.

\section{Energy metabolism}

The efficient regulation of energy homeostasis is an essential factor for an organism's survival. It comprises a range of different processes, including energy uptake (i.e. eating), storage (mostly as lipids, glycogen or tissue protein), and expenditure (energy usage for biosynthetic processes, heat production or locomotion). Energy is taken up in the form of macronutrients - carbohydrates, fat, or protein. In most species, nutrient ingestion follows a strict circadian rhythm, and several clock genes have been shown to be involved in the regulation of metabolic homeostasis. Clock mutant mice show blunted diurnal activity rhythms resulting in elevated food intake during the usual resting phase (day) and less ingestion during the active phase (night). These mice become hyperphagic and obese [151]. Another study showed that daytime high fat diet (HFD) in mice leads to a significant higher weight gain than nighttime HFD [3]. This is a possible explanation for the clock mutants' obese phenotype. Similarly, Per 2 mutant mice show arrhythmic feeding behavior and eat significantly more under (HFD) conditions. These effects are based on a decreased level of alpha melanocyte-stimulating hormone $(\alpha-\mathrm{MSH})$, a well-known appetite suppressor, at the beginning of the light phase. Constant administration of $\alpha-\mathrm{MSH}$ to Per 2 mutants leads to reduced food uptake, revealing $\alpha$-MSH as a direct target of the clock gene Per2, independent of rhythmicity [167]. Interestingly, some of these effects are also seen after sleep restriction in rodents and in humans. In the latter, a restriction of sleep time to $4 \mathrm{~h}$ for only a few consecutive nights is enough to significantly increase appetite [139, 140]. Rats that are kept awake for 2 weeks using the classic discover-water technique show hyperphagy - although they lose weight under these conditions [119]. Of note, a number of other animal studies failed to confirm an increase in food uptake in response to sleep restriction, indicating that small variations in experimental procedures may have a significant impact on these processes [9, 170]. A straight-forward mechanistic explanation for a sleep-loss-mediated increase in energy uptake remains elusive. It was suggested that a temporal deregulation of peripheral orexigenic and anorexigenic hormones could underlie this effect. The most promising candidates are the gastrointestinal peptide ghrelin $[29,73]$ and the adipokine leptin secreted by white adipocytes [89]. In humans as well as in animals, sleep restriction or total sleep deprivation cause significant decreases in circulating leptin and increased ghrelin, thus promoting appetite and hunger [11, 44, 140, 145]. Human leptin plasma levels are partially dependent on meal time [128] and also on the circadian time and sleep state. Under un-stressed and constant feeding conditions, leptin shows a marked nocturnal rise in humans [128]. When sleeping time is shifted by $8 \mathrm{~h}$, leptin levels are differentially regulated by both the circadian system and sleep, resulting in a short period rhythm with peaks in the night and around mid-sleep phase [136]. In contrast, the diurnal expression of ghrelin seems not to be directly clock-regulated. Under ad libitum feeding conditions ghrelin shows a bimodal rhythm with peaks in the afternoon and towards the end of the dark phase in rats, correlating with gastric emptying and filling [103]. However, in humans, sleep triggers ghrelin release during night. Comparable to rats, humans also show a bimodal ghrelin rhythm with one peak in the afternoon and one peak during night. The peak during night is absent when test persons were sleep deprived [40]. Ghrelin signaling seems to have a strong influence on the circadian system. In cultured mouse brain slices containing the SCN, ghrelin administration increases firing rate of individual SCN neurons. Ghrelin receptor activation phase shifts $\mathrm{SCN}$ bioluminescence rhythms in culture and resets locomotor activity rhythms in intact mice [168]. Another agent connecting the circadian system, sleep, and food uptake is the neuropeptide orexin (or hypocretin/HCRT). Its two isoforms, orexin A and B, are exclusively expressed in neurons of the lateral hypothalamus. Both have potent wake-promoting effects and at the same time stimulate food intake [126]. Orexin release is circadian clock-controlled and Hcrt transcription rhythms are abolished in Clock mutant mice [151]. The SCN directly innervates orexigenic neurons [104]. Under starvation conditions, the sleep duration of rats is shortened [28], while sleep deprivation increases energy uptake [119]. The orexin system constitutes a potential candidate linking both processes. 
Similar to food uptake, energy metabolism is influenced by the circadian system and sleep. Both sleep and clock disruptions have strong effects on glucose and lipid metabolism. Several clock gene mutant mouse strains show phenotypes resembling aspects of the type II diabetes pathology. The overexpression of mutant Cryl protein results in polydipsia, polyuria, and hyperglycemia [109]. Clock mutant mice show hyperglycemia and hypoinsulinemia [151]. Moreover, Clock mutant and Bmal1 ${ }^{-/}$mice exhibit impaired glucose liberation from the liver. Under HFD conditions, these mice show deficient insulin regulation and beta cell function in the pancreas [125]. A liver-specific deletion of Bmall promotes hypoglycemia and deregulated expression of genes involved in glucose metabolism, such as phosphoenolpyruvate carboxykinase $1(P c k 1)$, glucokinase $(G c k)$, and glucose-6-phosphate translocase 1 (G6pt1/Slc37a4) [79]. The fact that polymorphisms in the Clock gene are associated with metabolic syndrome in man and that several Bmall haplotypes in rats are connected to type II diabetes underlines the connection between circadian genes and metabolism [130, 163]. CCG mutations can also cause diabetic symptoms. Nocturnin is a clockcontrolled deadenylase involved in post-transcriptional regulation of gene expression. Loss of Nocturnin (Ccrnl4) has strong effects on insulin sensitivity and glucose tolerance [53]. Other examples are the orphan nuclear receptor peroxisome proliferator-activated receptor $\alpha$ (Ppara) and tumor necrosis factor alpha (TNF- $\alpha$ ) $[54,108]$. Strikingly similar effects on metabolism have been attributed to sleep (or the lack thereof). The global trend towards shorter sleep times during the last decades was suggested as one of the factors underlying the alarming increase in the prevalence of the metabolic syndrome and type II diabetes [52, 106]. In line with this, poor sleep quality is a risk factor for type II diabetes [146]. Experimentally, restriction of sleep to $4 \mathrm{~h}$ per night for less than a week increases blood glucose levels while at the same time decreasing insulin sensitivity [139], suggesting that a chronic reduction of sleep time raises the risk of developing diabetes.

Other processes associated with clock gene function are lipid metabolism in adipocytes and energy expenditure in the muscles. Clock mutant mice suffer from hyperlipidemia [151], and Bmal1 is necessary for adipocyte differentiation from mouse embryonic fibroblast cultures. Restoration of BMAL1-expression in Bmal1-deficient 3T3-L1 precursor cells rescues adipogenesis. A treatment with PPAR $\gamma$ ligands reconstitutes the differentiation potential of these cells. In addition, many other lipid metabolism-related genes, like $a P 2, S R E B P-1 \alpha$, and perilipin, are effected by Bmall restoration, indicating that all these genes are direct targets of Bmall [134]. Interestingly, Bmall ${ }^{-/}$mice exhibit no alterations in body weight gain under a variety of diet conditions. However, a possible obesity phenotype in these animals might be confounded by their premature aging [74]. The Bmall regulator ROR $\alpha$ promotes fatty acid oxidation via its targets, caveolin-3 and CPT-1, lipogenesis, and lipid storage in skeletal muscles [82], while Nocturnindeficient mice show resistance to diet-induced obesity [53]. Although the molecular mechanisms are less well understood, several studies suggest that shortened sleep also has a strong influence on lipid metabolism. A chronic lack of sleep, either shortened sleep time or poor sleep quality, is a strong risk factor for obesity and the development of the metabolic syndrome $[49,69]$. In a large longitudinal study on nurses, Patel et al. [112] observed a cross-sectional Ushaped association between sleep duration and body mass index (BMI) development over several years. It was suggested that sleep effects on lipid metabolism are mediated by endocrine factors, such as cortisol, prolactin, or insulin, as well as by sympathetic hyperactivity, which had previously been linked to obesity and insulin resistance. In this manner, sleep restriction represents a minor form of chronic stress, thus activating the sympathicus and elevating epinephrine and norepinephrine secretion from the adrenal medulla. In line with this, sympathetic activation and catecholamine administration inhibit leptin expression and secretion [137], increase free fatty acid levels [66], and decrease insulin sensitivity [94]. Restricted sleep and sleep deprivation elevate glucocorticoid levels [85], further promoting visceral fat deposition and insulin resistance [122].

Several endocrine factors have been suggested as potential modulators of clock and sleep regulated aspects of energy metabolism, including adrenal glucocorticoids, pituitary hormones, as well as the "night hormone" melatonin. In most mammals and birds, melatonin is produced in the pineal gland during the night. The pineal receives direct and indirect signals from the SCN and is, therefore, rhythmically locked to the circadian master clock. Bi-directional links have been described between melatonin production and the regulation of glucose metabolism. In diabetic GotoKakizaki (GK) rats, melatonin levels are significantly reduced, while melatonin receptor expression in the pancreas is increased [113]. In line with this, melatonin signaling has a strong influence on insulin secretion from the pancreatic beta cells [102].

\section{Neuronal plasticity}

Clock gene-sleep interactions have also been reported in the context of neuronal plasticity and learning processes [51]. These include both short and long-term memory 
(LTM) formation and recall. The latter is thought to depend primarily on hippocampal long-term potentiation (LTP), a form of synaptic plasticity [96]. LTP efficiency is time-ofday dependent in the hippocampus and in the $\operatorname{SCN}[10,22$, 107]. Several studies show that circadian core clock genes are involved in long-term memory formation. Mutations of Npas 2 cause impaired LTM in a fear conditioning paradigm [50]. Several clock output factors also have a role in memory formation. Inhibition of melatonin or deletion of the gene encoding the SCN-secreted peptide vasoactive intestinal polypeptide (VIP) disrupts memory formation $[23,159]$. These phenotypes are often connected to cAMP signaling $[158,169]$. MAPK phosphorylation and cAMP and CREB phosphorylation are clock gene controlled in the hippocampus. The nadir of these events corresponds to the time when the strongest learning deficits are observed and pharmacological inhibition of MAPK phosphorylation during a learning task impairs memory formation [41]. Like memory formation, memory recall is under direct influence of clock genes. Cry $1 / 2^{-/-}$mice show normal spatial memory and perform well in simple avoidance tasks. They are, however, unable to efficiently learn in a more complex time-place context [153]. Similar findings were reported from Per2 mutant animals [160]. In a foodrewarded hippocampus-dependent spatial memory task (eight-arm radial maze) [129], $\mathrm{Perl}^{-/-}$mice fail more frequently than wild-type littermates [70]. Various publications show strong influences of sleep on memory. For instance, individuals carrying homozygous $\mathrm{Per} 3^{5 / 5}$ alleles exhibit an interesting connection among their clock genotype, sleep, and regional brain response patterns to an executive task. In contrast to $P E R 3^{4 / 4}$ participants, sleep deprivation correlates to changes in brain activity only in PER $3^{5 / 5}$ participants [154].

Although the influence of sleep on memory processes has primarily been studied in a neurophysiological rather than in a molecular context, there are several common features of sleep and clock gene impact on memory processes. The hypothesis that sleep has a positive influence on memory formation is not new [68]. Numerous studies provide evidence that both declarative and procedural memory processes benefit from proper sleep $[95,138]$. Even very short naps of a few minutes have been shown to improve declarative and procedural memory formation and recall $[77,78,99$, 150]. Re-entrainment of sleep patterns can restore cognitive functions either in transgenic mice carrying the Huntington's disease mutation [111] or in elderly patients showing symptoms of dementia [121]. However, until now, it is not fully clarified whether sleep has, like clock gene function, an influence on the formation of LTP. LTP can occur during REM sleep [15], and REM deprivation impairs LTP in the rat hippocampus [123].
On the other hand, REM deprivation does not necessary lead to disturbed memory formation [118]. Total sleep deprivation can lead to problems in learning [32], but this effect seems to be highly dependent on the subjects' general cognitive capacity [33]. In animals, current sleep deprivation protocols always include a certain stress component, which in itself can interfere with memory formation [63].

\section{Immune functions}

Similar to the brain, the immune system acts as a bidirectional interface between the organism and its environment. From a more general perspective, it also functions in a very similar way in terms of detection of, reaction to, and memorization of information. Sleep has a strong influence on the immune system and vice versa [93]. Inflammation state affects sleep time and quality in animals and humans $[16,30,38]$, while sleep restriction leads to higher mortality rates upon infection or sepsis [42, 43, 149]. Several immune parameters show circadian rhythmicity in the blood of humans and other mammals [80]. Clock gene expression is rhythmic in peripheral leukocytes [5]. Moreover, the secretion of important neuroendocrine immune modulators is under circadian as well as under sleep control. The activities of the hypothalamus pituitary adrenal (HPA) axis and the sympathetic nervous system, both stress activated, are influenced by the circadian system and sleep. In arrhythmic Per $2 / C r y 1$ double mutant mice, HPA axis regulation is strongly affected. The responsiveness of the adrenal to adrenocorticotropin stimulation and, thus, the production of glucocorticoids are regulated by adrenocortical circadian clocks [110]. Humans show elevated cortisol and norepinephrine levels in response to sleep deprivation while epinephrine levels become arrhythmic [80]. These effects will likely give rise to changes observed in leukocyte production. For leukocytes, strong diurnal rhythms have been reported [14, 144], some of which seem to directly respond to cortisol secretion during night time and to epinephrine during the day [36, 37]. Moreover, sleep loss affects levels of lymphocytes, monocytes, natural killer (NK) cells, and T-cell proliferation in humans [12, 14, 35, 97]. Not only the appearance of immune cells is circadian as well as sleep-controlled but also the levels of several cytokines [93]. While interleukin (IL)-6 production seems to primarily depend on clock function, rhythmic TNF- $\alpha$, IL-10, and IL12 release from monocytes as well as IL-12 production by dendritic cells depend critically on sleep-wake conditions [80].

Circadian rhythm disruption has been shown to severely weaken the immune system. Mice exposed to four consecutive weekly 6-h phase advances of the light/dark 
schedule (a repetitive jet lag paradigm) show increased mortality in response to lipopolysaccharide (LPS) injection [20]. Likewise, the clock gene Per2 has been identified to play a direct role in the activation of macrophages by controlling the expression of interferon- $\gamma$ (IFN- $\gamma$ ), a macrophage activating factor, in the mouse spleen [7]. The same is true for the natural killer (NK) cell receptors LY49C and NKG2D [91]. NK cell-specific knockdown of Per2 leads to decreased protein levels of the immune factors granzyme B and perforin in rats, but not of IFN- $\gamma$ [8]. Interestingly, LPS administration in Per2-deficient mice provokes attenuated immune responses and yields considerable higher survival rates than in wild-type animals [88]. Bmall ${ }^{-/-}$mice show significantly reduced levels of B cells in peripheral blood, spleen and bone marrow [143]. $P E R 3^{5 / 5}$ individuals show elevated IL-6 concentrations compared to those with the $P E R^{4 / 4}$ genotype [55]. As for metabolism, melatonin secretion might be one of the messengers linking sleep, circadian system, and immune function. Chemical inhibition of melatonin secretion leads to decreased antibody responses in mice. This effect is reversed by melatonin administration [92]. Melatonin further promotes the production of macrophage and granulocyte progenitor cells and affects the production of cytokines, such as IL-1, IL-2, IL-6, IL-12, TGF- $\beta$, M-CSF, and TNF- $\alpha$ [141]. Vice versa, cytokines might have influence on clock gene expression. TNF- $\alpha$ and IL- $1 \beta$ suppress the expression of Per1-3 and Rev-erb $\alpha$ in fibroblasts and liver of mice [21] in a p38 MAP kinase and calcium-dependent way [114]. This impairment of clock genes might lead to increased fatigue seen after infections.

Both sleep deprivation and clock mutations deregulate the production of pro-inflammatory cytokines, and low-grade systemic inflammation is a known pathological component of obesity and metabolic syndrome [64, 98]. Moreover, the production of IL-1 is increased in humans with self-reported poor sleep quality. Remarkably, this correlation of sleep debt and IL-1 levels does not apply for obese humans (BMI $\geq 30)$ [115]. Elderly people with metabolic syndrome and systemic inflammation show a higher risk of cognitive impairment compared with those without metabolic syndrome or with metabolic syndrome but without inflammation [166]. This leads to the suggestion of a direct connection between sleep, circadian clock genes, metabolism, cognition, and the immune system.

\section{Conclusions}

Although it seems clear that sleep and clock genes have strong influences on various physiological processes, it is often technically difficult to disentangle clock gene, circadian rhythm, and sleep-specific influences because of their mutual dependency and because only little so far is known about the underlying mechanisms and circuits (summarized in Fig. 2). While, in this paper, we have independently evaluated the impact of both processes, it is well conceivable that some of the physiological functions of one might be mediated via regulation of the other, i.e., sleep regulates clocks regulate physiology or clocks regulate sleep regulate physiology - and vice versa. While tremendous progress has been made in deciphering the molecular machinery of circadian clocks, little is still known of how different tissue oscillators communicate with each other to synchronize behavior and physiology. Even less understood are the processes underlying sleep. In fact, the tight interaction between clocks and sleep processes and the surprisingly strong effect that clock gene mutations have on both processes $\mathrm{c}$ and $\mathrm{s}$ might provide new inroads into mapping the sleep circuitry of the brain and into identifying molecular substrates of sleep within neuronal cells. With the advent of conditional genetics in mice and functional brain imaging techniques in humans, new tools have been developed to more specifically address these issues in the living organism. On the other hand, improved experimental paradigms need to be developed to resolve some of the conflicting findings from animal and human studies. The pathological long-term effects of the progressing sleeplessness and circadian desynchrony of modern societies will be in the focus of future research. A better understanding of the underlying mechanisms might well become a key for advanced therapeutic strategies against some of the most pressing health issues such as diabetes and neuropsychiatric disorders.

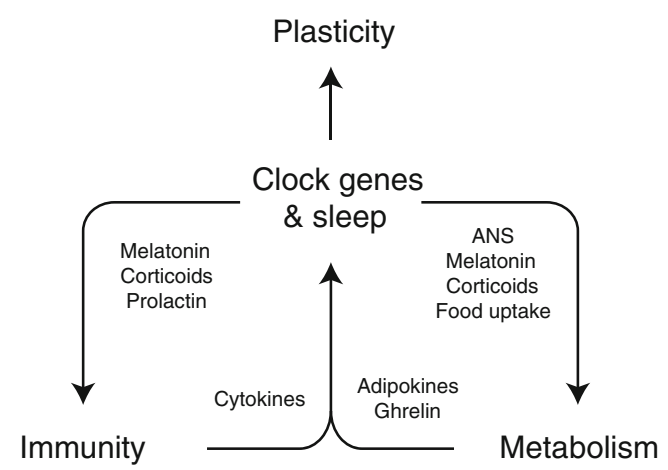

Fig. 2 Interaction of sleep and clocks in the regulation of cognitive and physiological processes. A reciprocal interactivity exists between sleep and circadian clock function. Both neuronal and blood-borne factors have been proposed to mediate clocksleep and sleep-physiology communication. On the other hand, peripheral humoral factors have been shown to provide feedback about the physiological state to sleep and clock regulatory circuits. The local regulation of physiology by tissue clocks may serve to integrate sleep state and timing signals at the cellular level. For details, see text 
Acknowledgments We would like to thank Johanna Barclay for critical reading of the manuscript. H.O. is an Emmy Noether fellow of the German Research Foundation (DFG). A.S. is supported by the Max Planck Society (MPG).

\section{References}

1. Allebrandt KV et al (2010) CLOCK gene variants associate with sleep duration in two independent populations. Biol Psychiatry 67 (11):1040-1047

2. Antoch MP et al (1997) Functional identification of the mouse circadian Clock gene by transgenic BAC rescue. Cell 89(4):655-667

3. Arble DM et al (2009) Circadian timing of food intake contributes to weight gain. Obesity (Silver Spring) 17 (11):2100-2102

4. Archer SN et al (2003) A length polymorphism in the circadian clock gene Per3 is linked to delayed sleep phase syndrome and extreme diurnal preference. Sleep 26(4):413-415

5. Archer SN et al (2008) Inter-individual differences in habitual sleep timing and entrained phase of endogenous circadian rhythms of BMAL1, PER2 and PER3 mRNA in human leukocytes. Sleep 31(5):608-617

6. Archer SN et al (2010) Polymorphism in the PER3 promoter associates with diurnal preference and delayed sleep phase disorder. Sleep 33(5):695-701

7. Arjona A, Sarkar DK (2006) The circadian gene mPer2 regulates the daily rhythm of IFN-gamma. J Interferon Cytokine Res 26 (9):645-649

8. Arjona A, Sarkar DK (2006) Evidence supporting a circadian control of natural killer cell function. Brain Behav Immun 20 (5):469-476

9. Balestrieri S, D'Onofrio G, Giuditta A (1980) Deprivation of paradoxical sleep. Effect on weight and nucleic acid content of liver and brain. Neurochem Res 5(12):1251-1264

10. Barnes CA et al (1977) Circadian rhythm of synaptic excitability in rat and monkey central nervous system. Science 197(4298):91-92

11. Bodosi B et al (2004) Rhythms of ghrelin, leptin, and sleep in rats: effects of the normal diurnal cycle, restricted feeding, and sleep deprivation. Am J Physiol Regul Integr Comp Physiol 287 (5):R1071-R1079

12. Bollinger $\mathrm{T}$ et al (2009) Sleep-dependent activity of T cells and regulatory T cells. Clin Exp Immunol 155(2):231-238

13. Borbely AA (1982) A two process model of sleep regulation. Hum Neurobiol 1(3):195-204

14. Born J et al (1997) Effects of sleep and circadian rhythm on human circulating immune cells. J Immunol 158(9):4454-4464

15. Bramham CR, Srebro B (1989) Synaptic plasticity in the hippocampus is modulated by behavioral state. Brain Res 493 (1):74-86

16. Bryant PA, Trinder J, Curtis N (2004) Sick and tired: does sleep have a vital role in the immune system? Nat Rev Immunol 4 (6):457-467

17. Bunger MK et al (2000) Mop3 is an essential component of the master circadian pacemaker in mammals. Cell 103 (7):1009-1017

18. Carpen JD et al (2005) A single-nucleotide polymorphism in the 5 -untranslated region of the hPER 2 gene is associated with diurnal preference. J Sleep Res 14(3):293-297

19. Carpen JD et al (2006) A silent polymorphism in the PER1 gene associates with extreme diurnal preference in humans. J Hum Genet 51(12):1122-1125
20. Castanon-Cervantes O et al (2010) Dysregulation of inflammatory responses by chronic circadian disruption. J Immunol 185 (10):5796-5805

21. Cavadini G et al (2007) TNF-alpha suppresses the expression of clock genes by interfering with E-box-mediated transcription. Proc Natl Acad Sci USA 104(31):12843-12848

22. Chaudhury D, Wang LM, Colwell CS (2005) Circadian regulation of hippocampal long-term potentiation. J Biol Rhythms 20(3):225-236

23. Chaudhury D et al (2008) Select cognitive deficits in vasoactive intestinal peptide deficient mice. BMC Neurosci 9:63

24. Cheng MY et al (2002) Prokineticin 2 transmits the behavioural circadian rhythm of the suprachiasmatic nucleus. Nature 417 (6887):405-410

25. Chung BY et al (2009) The GABA(A) receptor RDL acts in peptidergic PDF neurons to promote sleep in Drosophila. Curr Biol 19(5):386-390

26. Cyran SA et al (2003) vrille, Pdp1, and dClock form a second feedback loop in the Drosophila circadian clock. Cell 112 (3):329-341

27. Daan S, Beersma DG, Borbely AA (1984) Timing of human sleep: recovery process gated by a circadian pacemaker. Am J Physiol 246(2 Pt 2):R161-R183

28. Danguir J, Nicolaidis S (1979) Dependence of sleep on nutrients' availability. Physiol Behav 22(4):735-740

29. Date $\mathrm{Y}$ et al (2000) Ghrelin, a novel growth hormone-releasing acylated peptide, is synthesized in a distinct endocrine cell type in the gastrointestinal tracts of rats and humans. Endocrinology 141(11):4255-4261

30. Deboer $\mathrm{T}$ et al (2003) Sleep states alter activity of suprachiasmatic nucleus neurons. Nat Neurosci 6(10):1086-1090

31. Deboer T, Detari L, Meijer JH (2007) Long term effects of sleep deprivation on the mammalian circadian pacemaker. Sleep 30 (3):257-262

32. Diekelmann S et al (2008) Sleep loss produces false memories. PLoS One 3(10):e3512

33. Diekelmann S, Born J, Wagner U (2010) Sleep enhances false memories depending on general memory performance. Behav Brain Res 208(2):425-429

34. Dijk DJ, Czeisler CA (1995) Contribution of the circadian pacemaker and the sleep homeostat to sleep propensity, sleep structure, electroencephalographic slow waves, and sleep spindle activity in humans. J Neurosci Off $\mathrm{J}$ Soc Neurosci $15(5 \mathrm{Pt}$ 1):3526-3538

35. Dimitrov $S$ et al (2007) Number and function of circulating human antigen presenting cells regulated by sleep. Sleep 30 (4):401-411

36. Dimitrov S et al (2009) Cortisol and epinephrine control opposing circadian rhythms in $\mathrm{T}$ cell subsets. Blood 113 (21):5134-5143

37. Dimitrov S, Lange T, Born J (2010) Selective mobilization of cytotoxic leukocytes by epinephrine. J Immunol 184(1):503-511

38. Drake CL et al (2000) Effects of an experimentally induced rhinovirus cold on sleep, performance, and daytime alertness. Physiol Behav 71(1-2):75-81

39. Dudley CA et al (2003) Altered patterns of sleep and behavioral adaptability in NPAS2-deficient mice. Science 301 (5631):379-383

40. Dzaja A et al (2004) Sleep enhances nocturnal plasma ghrelin levels in healthy subjects. Am J Physiol Endocrinol Metab 286 (6):E963-E967

41. Eckel-Mahan KL et al (2008) Circadian oscillation of hippocampal MAPK activity and cAmp: implications for memory persistence. Nat Neurosci 11(9):1074-1082

42. Everson CA (1993) Sustained sleep deprivation impairs host defense. Am J Physiol 265(5 Pt 2):R1148-R1154 
43. Everson CA, Toth LA (2000) Systemic bacterial invasion induced by sleep deprivation. Am J Physiol Regul Integr Comp Physiol 278(4):R905-R916

44. Everson CA, Crowley WR (2004) Reductions in circulating anabolic hormones induced by sustained sleep deprivation in rats. Am J Physiol Endocrinol Metab 286(6):E1060-E1070

45. Franken $P$ et al (2000) The transcription factor DBP affects circadian sleep consolidation and rhythmic EEG activity. J Neurosci 20(2):617-625

46. Franken $P$ et al (2006) NPAS2 as a transcriptional regulator of non-rapid eye movement sleep: genotype and sex interactions. Proc Natl Acad Sci USA 103(18):7118-7123

47. Franken P, Dijk DJ (2009) Circadian clock genes and sleep homeostasis. Eur J Neurosci 29(9):1820-1829

48. Gallego M, Virshup DM (2007) Post-translational modifications regulate the ticking of the circadian clock. Nat Rev Mol Cell Biol 8(2):139-148

49. Gangwisch JE et al (2005) Inadequate sleep as a risk factor for obesity: analyses of the NHANES I. Sleep 28(10):1289-1296

50. Garcia JA et al (2000) Impaired cued and contextual memory in NPAS2-deficient mice. Science 288(5474):2226-2230

51. Gerstner JR (2010) The aging clock: to 'BMAL'icious toward learning and memory. Aging 2(5):251-254

52. Gottlieb DJ et al (2005) Association of sleep time with diabetes mellitus and impaired glucose tolerance. Arch Intern Med 165 (8):863-867

53. Green CB et al (2007) Loss of Nocturnin, a circadian deadenylase, confers resistance to hepatic steatosis and diet-induced obesity. Proc Natl Acad Sci USA 104(23):9888-9893

54. Grimble RF (2002) Inflammatory status and insulin resistance. Curr Opin Clin Nutr Metab Care 5(5):551-559

55. Guess J et al (2009) Circadian disruption, Per3, and human cytokine secretion. Integr Cancer Ther 8(4):329-336

56. Hardin PE (2005) The circadian timekeeping system of Drosophila. Curr Biol 15(17):R714-R722

57. Hardin PE, Hall JC, Rosbash M (1990) Feedback of the Drosophila period gene product on circadian cycling of its messenger RNA levels. Nature 343(6258):536-540

58. Harmar AJ et al (2002) The VPAC(2) receptor is essential for circadian function in the mouse suprachiasmatic nuclei. Cell 109 (4):497-508

59. He Y et al (2009) The transcriptional repressor DEC2 regulates sleep length in mammals. Science 325(5942):866-870

60. Hendricks JC et al (2000) Rest in Drosophila is a sleep-like state. Neuron 25(1):129-138

61. Hendricks JC et al (2003) Gender dimorphism in the role of cycle (BMAL1) in rest, rest regulation, and longevity in Drosophila melanogaster. J Biol Rhythms 18(1):12-25

62. Honma S et al (2002) Dec1 and Dec2 are regulators of the mammalian molecular clock. Nature 419(6909):841-844

63. Horne JA, McGrath MJ (1984) The consolidation hypothesis for REM sleep function: stress and other confounding factors-a review. Biol Psychol 18(3):165-184

64. Hotamisligil GS, Erbay E (2008) Nutrient sensing and inflammation in metabolic diseases. Nat Rev Immunol 8 (12):923-934

65. Hu WP et al (2007) Altered circadian and homeostatic sleep regulation in prokineticin 2-deficient mice. Sleep 30(3):247-256

66. Hucking $\mathrm{K}$ et al (2003) Burst-like control of lipolysis by the sympathetic nervous system in vivo. J Clin Invest 111 (2):257-264

67. Ibuka N, Nihonmatsu I, Sekiguchi S (1980) Sleep-wakefulness rhythms in mice after suprachiasmatic nucleus lesions. Waking Sleeping 4(2):167-173

68. Jenkins JG, Dallenbach KM (1924) Obliviscence during sleep and waking. Am J Psychol 35:605-612
69. Jennings JR et al (2007) Self-reported sleep quality is associated with the metabolic syndrome. Sleep 30(2):219-223

70. Jilg A et al (2010) Temporal dynamics of mouse hippocampal clock gene expression support memory processing. Hippocampus 20(3):377-388

71. King DP et al (1997) Positional cloning of the mouse circadian clock gene. Cell 89(4):641-653

72. Ko HW, Jiang J, Edery I (2002) Role for Slimb in the degradation of Drosophila Period protein phosphorylated by Doubletime. Nature 420(6916):673-678

73. Kojima M et al (1999) Ghrelin is a growth-hormone-releasing acylated peptide from stomach. Nature 402(6762):656-660

74. Kondratov RV et al (2006) Early aging and age-related pathologies in mice deficient in BMAL1, the core componentof the circadian clock. Genes Dev 20(14):1868-1873

75. Konopka RJ, Benzer S (1971) Clock mutants of Drosophila melanogaster. Proc Natl Acad Sci USA 68(9):2112-2116

76. Kopp C et al (2002) Homeostatic sleep regulation is preserved in mPer1 and mPer2 mutant mice. Eur J Neurosci 16(6):1099-1106

77. Korman M et al (2007) Daytime sleep condenses the time course of motor memory consolidation. Nat Neurosci 10(9):1206-1213

78. Lahl O et al (2008) An ultra short episode of sleep is sufficient to promote declarative memory performance. J Sleep Res 17(1):3-10

79. Lamia KA, Storch KF, Weitz CJ (2008) Physiological significance of a peripheral tissue circadian clock. Proc Natl Acad Sci USA 105(39):15172-15177

80. Lange T, Dimitrov S, Born J (2010) Effects of sleep and circadian rhythm on the human immune system. Ann N Y Acad Sci 1193:48-59

81. Laposky A et al (2005) Deletion of the mammalian circadian clock gene BMAL1/Mop3 alters baseline sleep architecture and the response to sleep deprivation. Sleep 28(4):395-409

82. Lau P et al (2004) RORalpha regulates the expression of genes involved in lipid homeostasis in skeletal muscle cells: caveolin-3 and CPT-1 are direct targets of ROR. J Biol Chem 279(35):36828-36840

83. Lee $\mathrm{C}$ et al (2001) Posttranslational mechanisms regulate the mammalian circadian clock. Cell 107(7):855-867

84. Lee ML, Swanson BE, de la Iglesia HO (2009) Circadian timing of REM sleep is coupled to an oscillator within the dorsomedial suprachiasmatic nucleus. Curr Biol 19(10):848-852

85. Leproult $\mathrm{R}$ et al (1997) Sleep loss results in an elevation of cortisol levels the next evening. Sleep 20(10):865-870

86. Li JD et al (2006) Attenuated circadian rhythms in mice lacking the prokineticin 2 gene. J Neurosci 26(45):11615-11623

87. Lim C et al (2007) Clockwork orange encodes a transcriptional repressor important for circadian-clock amplitude in Drosophila. Curr Biol 17(12):1082-1089

88. Liu J et al (2006) The circadian clock Period 2 gene regulates gamma interferon production of NK cells in host response to lipopolysaccharide-induced endotoxic shock. Infect Immun 74 (8):4750-4756

89. Lonnqvist $\mathrm{F}$ et al (1995) Overexpression of the obese (ob) gene in adipose tissue of human obese subjects. Nat Med 1(9):950-953

90. Lopez-Molina L et al (1997) The DBP gene is expressed according to a circadian rhythm in the suprachiasmatic nucleus and influences circadian behavior. EMBO J 16(22):6762-6771

91. Luo $Y$ et al (2009) Expression profiling reveals a positive regulation by mPer2 on circadian rhythm of cytotoxicity receptors: Ly49C and Nkg2d. Chronobiol Int 26(8):1514-1544

92. Maestroni GJ, Conti A, Pierpaoli W (1986) Role of the pineal gland in immunity. Circadian synthesis and release of melatonin modulates the antibody response and antagonizes the immunosuppressive effect of corticosterone. J Neuroimmunol 13(1):19-30 
93. Majde JA, Krueger JM (2005) Links between the innate immune system and sleep. J Allergy Clin Immunol 116(6):1188-1198

94. Marangou AG et al (1988) Hormonal effects of norepinephrine on acute glucose disposal in humans: a minimal model analysis. Metabolism 37(9):885-891

95. Marshall L, Born J (2007) The contribution of sleep to hippocampus-dependent memory consolidation. Trends Cogn Sci 11(10):442-450

96. Martin SJ, Grimwood PD, Morris RG (2000) Synaptic plasticity and memory: an evaluation of the hypothesis. Annu Rev Neurosci 23:649-711

97. Matsumoto $\mathrm{Y}$ et al (2001) Total sleep deprivation induces an acute and transient increase in NK cell activity in healthy young volunteers. Sleep 24(7):804-809

98. Maury E, Ramsey KM, Bass J (2010) Circadian rhythms and metabolic syndrome: from experimental genetics to human disease. Circ Res 106(3):447-462

99. Mednick S, Nakayama K, Stickgold R (2003) Sleep-dependent learning: a nap is as good as a night. Nat Neurosci 6(7):697-698

100. Mistlberger RE et al (1983) Recovery sleep following sleep deprivation in intact and suprachiasmatic nuclei-lesioned rats. Sleep 6(3):217-233

101. Mitsui $S$ et al (2001) Antagonistic role of E4BP4 and PAR proteins in the circadian oscillatory mechanism. Genes Dev 15 (8):995-1006

102. Mulder H et al (2009) Melatonin receptors in pancreatic islets: good morning to a novel type 2 diabetes gene. Diabetologia 52 (7):1240-1249

103. Murakami N et al (2002) Role for central ghrelin in food intake and secretion profile of stomach ghrelin in rats. J Endocrinol 174 (2):283-288

104. Nambu T et al (1999) Distribution of orexin neurons in the adult rat brain. Brain Res 827(1-2):243-260

105. Naylor E et al (2000) The circadian clock mutation alters sleep homeostasis in the mouse. J Neurosci 20(21):8138-8143

106. Nilsson PM et al (2004) Incidence of diabetes in middleaged men is related to sleep disturbances. Diabetes Care 27 (10):2464-2469

107. Nishikawa Y, Shibata S, Watanabe S (1995) Circadian changes in long-term potentiation of rat suprachiasmatic field potentials elicited by optic nerve stimulation in vitro. Brain Res 695 (2):158-162

108. Oishi K, Shirai H, Ishida N (2005) CLOCK is involved in the circadian transactivation of peroxisome-proliferator-activated receptor alpha (PPARalpha) in mice. Biochem J 386 (Pt 3):575-581

109. Okano $\mathrm{S}$ et al (2009) Unusual circadian locomotor activity and pathophysiology in mutant CRY1 transgenic mice. Neurosci Lett 451(3):246-251

110. Oster $\mathrm{H}$ et al (2006) The circadian rhythm of glucocorticoids is regulated by a gating mechanism residing in the adrenal cortical clock. Cell Metab 4(2):163-173

111. Pallier PN et al (2007) Pharmacological imposition of sleep slows cognitive decline and reverses dysregulation of circadian gene expression in a transgenic mouse model of Huntington's disease. J Neurosci Off J Soc Neurosci 27 (29):7869-7878

112. Patel SR et al (2004) A prospective study of sleep duration and mortality risk in women. Sleep 27(3):440-444

113. Peschke E et al (2006) Diabetic Goto Kakizaki rats as well as type 2 diabetic patients show a decreased diurnal serum melatonin level and an increased pancreatic melatonin-receptor status. J Pineal Res 40(2):135-143

114. Petrzilka S et al (2009) Clock gene modulation by TNF-alpha depends on calcium and p38 MAP kinase signaling. J Biol Rhythms 24(4):283-294
115. Prather AA et al (2009) Normative variation in self-reported sleep quality and sleep debt is associated with stimulated proinflammatory cytokine production. Biol Psychol 82(1):12-17

116. Preitner N et al (2002) The orphan nuclear receptor REVERBalpha controls circadian transcription within the positive limb of the mammalian circadian oscillator. Cell 110(2):251-260

117. Price JL et al (1998) Double-time is a novel Drosophila clock gene that regulates PERIOD protein accumulation. Cell 94 (1):83-95

118. Rasch B et al (2009) Pharmacological REM sleep suppression paradoxically improves rather than impairs skill memory. Nat Neurosci 12(4):396-397

119. Rechtschaffen A, Bergmann BM (1995) Sleep deprivation in the rat by the disk-over-water method. Behav Brain Res 69(1-2):55-63

120. Reppert SM, Weaver DR (2002) Coordination of circadian timing in mammals. Nature 418(6901):935-941

121. Riemersma-van der Lek RF et al (2008) Effect of bright light and melatonin on cognitive and noncognitive function in elderly residents of group care facilities: a randomized controlled trial. JAMA 299(22):2642-2655

122. Roberge $\mathrm{C}$ et al (2007) Adrenocortical dysregulation as a major player in insulin resistance and onset of obesity. Am J Physiol Endocrinol Metab 293(6):E1465-E1478

123. Romcy-Pereira R, Pavlides C (2004) Distinct modulatory effects of sleep on the maintenance of hippocampal and medial prefrontal cortex LTP. Eur J Neurosci 20(12):3453-3462

124. Rossner MJ et al (2008) Disturbed clockwork resetting in Sharp-1 and Sharp-2 single and double mutant mice. PLoS One 3(7):e2762

125. Rudic RD et al (2004) BMAL1 and CLOCK, two essential components of the circadian clock, are involved in glucose homeostasis. PLoS Biol 2(11):e377

126. Sakurai T (2005) Roles of orexin/hypocretin in regulation of sleep/wakefulness and energy homeostasis. Sleep Med Rev 9 (4):231-241

127. Sato TK et al (2004) A functional genomics strategy reveals Rora as a component of the mammalian circadian clock. Neuron 43(4):527-537

128. Schoeller DA et al (1997) Entrainment of the diurnal rhythm of plasma leptin to meal timing. J Clin Invest 100(7):1882-1887

129. Schwegler H, Crusio WE, Brust I (1990) Hippocampal mossy fibers and radial-maze learning in the mouse: a correlation with spatial working memory but not with non-spatial reference memory. Neuroscience 34(2):293-298

130. Scott EM, Carter AM, Grant PJ (2008) Association between polymorphisms in the Clock gene, obesity and the metabolic syndrome in man. Int J Obes (Lond) 32(4):658-662

131. Shaw PJ et al (2000) Correlates of sleep and waking in Drosophila melanogaster. Science 287(5459):1834-1837

132. Shaw PJ et al (2002) Stress response genes protect against lethal effects of sleep deprivation in Drosophila. Nature 417 (6886):287-291

133. Sheward WJ et al (2010) Circadian control of mouse heart rate and blood pressure by the suprachiasmatic nuclei: behavioral effects are more significant than direct outputs. PLoS One 5(3):e9783

134. Shimba S et al (2005) Brain and muscle Arnt-like protein-1 (BMAL1), a component of the molecular clock, regulates adipogenesis. Proc Natl Acad Sci USA 102(34):12071-12076

135. Shiromani PJ et al (2004) Sleep rhythmicity and homeostasis in mice with targeted disruption of mPeriod genes. Am J Physiol Regul Integr Comp Physiol 287(1):R47-R57

136. Simon C et al (1998) Circadian and ultradian variations of leptin in normal man under continuous enteral nutrition: relationship to sleep and body temperature. J Clin Endocrinol Metab 83 (6):1893-1899 
137. Slieker LJ et al (1996) Regulation of expression of ob mRNA and protein by glucocorticoids and cAMP. J Biol Chem 271 (10):5301-5304

138. Smith C (2001) Sleep states and memory processes in humans: procedural versus declarative memory systems. Sleep Med Rev 5 (6):491-506

139. Spiegel K, Leproult R, Van Cauter E (1999) Impact of sleep debt on metabolic and endocrine function. Lancet 354 (9188):1435-1439

140. Spiegel K et al (2004) Brief communication: sleep curtailment in healthy young men is associated with decreased leptin levels, elevated ghrelin levels, and increased hunger and appetite. Ann Intern Med 141(11):846-850

141. Srinivasan V et al (2005) Melatonin, immune function and aging. Immun Ageing 2:17

142. Stanewsky R et al (1998) The cryb mutation identifies cryptochrome as a circadian photoreceptor in Drosophila. Cell 95(5):681-692

143. Sun Y et al (2006) MOP3, a component of the molecular clock, regulates the development of B cells. Immunology 119 (4):451-460

144. Suzuki S et al (1997) Circadian rhythm of leucocytes and lymphocytes subsets and its possible correlation with the function of the autonomic nervous system. Clin Exp Immunol 110(3):500-508

145. Taheri $\mathrm{S}$ et al (2004) Short sleep duration is associated with reduced leptin, elevated ghrelin, and increased body mass index. PLoS Med 1(3): 62

146. Tasali E, Mokhlesi B, Van Cauter E (2008) Obstructive sleep apnea and type 2 diabetes: interacting epidemics. Chest 133 (2):496-506

147. Tobler I, Borbely AA, Groos G (1983) The effect of sleep deprivation on sleep in rats with suprachiasmatic lesions. Neurosci Lett 42(1):49-54

148. Toh KL et al (2001) An hPer2 phosphorylation site mutation in familial advanced sleep phase syndrome. Science 291 (5506):1040-1043

149. Toth LA, Tolley EA, Krueger JM (1993) Sleep as a prognostic indicator during infectious disease in rabbits. Proc Soc Exp Biol Med 203(2):179-192

150. Tucker MA et al (2006) A daytime nap containing solely non-REM sleep enhances declarative but not procedural memory. Neurobiol Learn Mem 86(2):241-247

151. Turek FW et al (2005) Obesity and metabolic syndrome in circadian Clock mutant mice. Science 308(5724):1043-1045

152. van der Horst GT et al (1999) Mammalian Cry1 and Cry2 are essential for maintenance of circadian rhythms. Nature 398 (6728):627-630

153. Van der Zee EA et al (2008) Circadian time-place learning in mice depends on Cry genes. Curr Biol 18(11):844-848
154. Vandewalle G et al (2009) Functional magnetic resonance imaging-assessed brain responses during an executive task depend on interaction of sleep homeostasis, circadian phase, and PER3 genotype. J Neurosci Off J Soc Neurosci 29 (25):7948-7956

155. Viola AU et al (2007) PER3 polymorphism predicts sleep structure and waking performance. Curr Biol 17(7):613-618

156. Vitaterna MH et al (1994) Mutagenesis and mapping of a mouse gene, Clock, essential for circadian behavior. Science 264 (5159):719-725

157. Vitaterna MH et al (1999) Differential regulation of mammalian period genes and circadian rhythmicity by cryptochromes 1 and 2. Proc Natl Acad Sci USA 96(21):12114-12119

158. von Gall C et al (1998) CREB in the mouse SCN: a molecular interface coding the phase-adjusting stimuli light, glutamate, PACAP, and melatonin for clockwork access. J Neurosci 18 (24):10389-10397

159. Wang LM et al (2005) Melatonin inhibits hippocampal long-term potentiation. Eur J Neurosci 22(9):2231-2237

160. Wang, LM et al. (2009) Expression of the circadian clock gene Period 2 in the hippocampus: possible implications for synaptic plasticity and learned behaviour. ASN Neuro. 1(3):e00012

161. Wisor JP et al (2002) A role for cryptochromes in sleep regulation. BMC Neurosci 3:20

162. Wisor JP et al (2008) Sleep deprivation effects on circadian clock gene expression in the cerebral cortex parallel electroencephalographic differences among mouse strains. J Neurosci 28 (28):7193-7201

163. Woon PY et al (2007) Aryl hydrocarbon receptor nuclear translocator-like (BMAL1) is associated with susceptibility to hypertension and type 2 diabetes. Proc Natl Acad Sci USA 104 (36): $14412-14417$

164. Xu Y et al (2005) Functional consequences of a CKIdelta mutation causing familial advanced sleep phase syndrome. Nature 434(7033):640-644

165. Xu Y et al (2007) Modeling of a human circadian mutation yields insights into clock regulation by PER2. Cell 128(1):59-70

166. Yaffe $\mathrm{K}$ et al (2004) The metabolic syndrome, inflammation, and risk of cognitive decline. JAMA 292(18):2237-2242

167. Yang $\mathrm{S}$ et al (2009) The role of mPer2 clock gene in glucocorticoid and feeding rhythms. Endocrinology 150 (5):2153-2160

168. Yannielli PC et al (2007) Ghrelin effects on the circadian system of mice. J Neurosci 27(11):2890-2895

169. Yin JC et al (1995) CREB as a memory modulator: induced expression of a dCREB2 activator isoform enhances long-term memory in Drosophila. Cell 81(1):107-115

170. Youngblood BD et al (1999) The effects of paradoxical sleep deprivation and valine on spatial learning and brain 5-HT metabolism. Physiol Behav 67(5):643-649 